\title{
AVAliaÇÃo do DESEnVOLVIMENTO EXPERIENCIAL DE PACIENTES COM PRÓTESE OCULAR: A FOCALIZAÇÃO NO ATENDIMENTO CLÍNICO
}

EXPERIENTIAL DEVELOPMENT ASSESSMENT OF PATIENTS WITH PROSTHETIC EYE: FOCUSING TECHNIQUE ON PSYCHOTHERAPY

EVALUACIÓN DEL DESARROLLO EXPERIENCIAL DE LOS PACIENTES CON PRÓTESIS OCULAR: LA FOCALIZACIÓN EN LA ATENCIÓN CLÍNICA

Marilia Souza da Silveira* Cristiano Mauro Assis Gomes**

\section{Resumo}

O presente estudo teve como objetivo verificar a eficácia da ferramenta de focalização utilizada em intervenção breve com pacientes em situação de prótese ocular. Foi realizado um estudo quasi-experimental de caso-controle com uma amostra de conveniência, composta por 10 participantes para o grupo experimental e oito para o controle. O Teste de Habilidade Experiencial (THE) foi utilizado para a coleta de dados em dois momentos em desenho de pré-teste/pós-teste. O grupo experimental vivenciou quatro sessóes terapêuticas de focalização. O tamanho do efeito da diferença de crescimento da média do grupo experimental e do grupo controle foi pequeno-moderado $(d=0,38 ; p=0,44)$. Implicaçóes dos resultados para a prática da psicologia clínica são discutidas.

Palavras-chave: focalização; prótese ocular; Teste de Habilidade Experiencial (THE).

\section{AbSTRACT}

The present study aimed to verify the effectiveness of the focusing therapy applied in brief intervention with patients in context of ocular prosthesis. The de-

\footnotetext{
* Universidade Federal de Minas Gerais, Belo Horizonte, MG, Brasil.

** Universidade Federal de Minas Gerais, Belo Horizonte, MG, Brasil.
} 
sign of case-control study included a convenience sample with 10 participants for the experimental group and eight participants for the control group. The data was collected by the Experiential Ability Test (EAT) on the pre-test and pos-test. The experimental group experienced four psychotherapy sessions guided by the focusing technique. The effect size of the difference in average growth in the experimental group and control group was small-moderate $(\mathrm{d}=0.38, \mathrm{p}=0.44)$. Implications for the practice of clinical psychology are discussed.

Keywords: focusing; ocular prosthesis; Experiential Ability Test (EAT).

\section{Resumen}

El presente estudio tuvo como objetivo verificar la eficacia de la herramienta de focalización utilizado en breves intervenciones con pacientes con la prótesis ocular. Se realizó un estudio cuasi-experimental de casos y controles con una muestra compuesta por 10 participantes en el grupo experimental y ocho en el de control. El Test de la Habilidade Experiencial (THE) se utilizó para recopilar datos en dos puntos, pré-teste/pós-teste. El grupo experimental experimentaron cuatro sesiones terapéuticas de la focalización. El tamaño del efecto de la diferencia en el crecimiento promedio del grupo experimental y el grupo de control fue pequeńa-moderada $(\mathrm{d}=0,38, \mathrm{p}=0,44)$. Se discuten las implicaciones de los resultados para la práctica de la psicología clínica.

Palabras clave: focalización; prótesis ocular; Test de la Habilidad Experiencial (THE).

\section{Introdução}

A ideia de prótese ocular, ou olho artificial, nasceu paralelamente ao desenvolvimento das artes plásticas, há 2500 anos a.C. (Resende, 1997). Antes disso, nas pinturas rupestres, a região ocular não era reproduzida, passando a ser observada em algumas pinturas e estátuas quando a importância da reprodução dos detalhes nas obras de artes tornou-se significativa. "A história relata que os antigos egípcios, com a preocupação de embelezar suas estátuas, confeccionavam olhos artificiais com ouro e pedras preciosas, o que certamente inspirou sua manifestação no vivo”. (Fonseca, 1987, p. 19).

Em seres humanos, a desconfiguração ocular era inicialmente camuflada com tapa-olho de seda ou metal, tendo a pintura das pálpebras e olho em sua superfície para efeito estético. Posteriormente, a prótese intracavitária foi desen- 
volvida em cerâmica e vidro por Ambroise Paré (1510-1590). Desde então, Paré passou a ser considerado o pai da prótese ocular que se industrializou na Alemanha, em 1835, com a colaboração de Ludwing Muller Uri (Fonseca, 1987). Como assoprador de vidro, Muller fabricava olhos para bonecas, passando a produzir olhos de vidro para seres humanos e levando o mercado de prótese ocular alemão à liderança na fabricação e exportação do produto. Frente à fragilidade do material e à necessidade de manter um estoque variado devido à coloração da íris e tamanho da prótese, os países importadores iniciaram suas buscas no aprimoramento da matéria-prima a fim de se tornarem independentes quanto à manufatura da prótese ocular. "Outro fator que estimulava a autossuficiência dizia respeito a um possível barateamento do produto, com o objetivo de beneficiar as camadas sociais menos favorecidas" (Fonseca, 1987, p. 25).

Diversos materiais foram testados na tentativa de aprimorar a prótese ocular, dentre eles, o alumínio, o marfim, o chifre e a celuloide, por serem leves e de fácil manipulação. No entanto, a técnica de confecção da prótese ocular não era compartilhada por seus autores, o que ocorreu apenas com a Segunda Guerra Mundial, frente à enorme ocorrência de traumatismos oculares e, também, a impossibilidade de os Estados Unidos receberem olhos artificiais vindos da Alemanha. $\mathrm{O}$ exército americano reuniu especialistas para publicarem trabalhos sobre técnicas de confecção de olhos artificiais individuais em acrílico, baseados em conhecimento de prótese dental (Resende, 1997, p. 131).

$\mathrm{Na}$ América do Sul, a prótese ocular pode ser considerada relativamente recente, já que as primeiras publicaçóes ocorreram em Santiago do Chile e $\mathrm{Ar}$ gentina, em 1949. No Brasil, os estudos e práticas deste recurso iniciaram-se no estado do Rio Grande do Sul protagonizados por Wilson Tupinambá, Eurico Kramer de Oliveira e Gaspar Soares Brandão, avançando para o norte do País com Gemaque Álvaro, e posteriormente para o nordeste com Antônio Gamboa Varela (Fonseca, 1987, p. 30).

Hoje, após um percurso histórico de constante aperfeiçoamento, a prótese ocular de melhor qualidade é realizada individualmente para cada cavidade anoftálmica, confeccionada em resina acrílica e pintada manualmente, de modo a copiar todas as características do olho remanescente do paciente, oferecendo um resultado estético satisfatório, além de contribuir significativamente no que diz respeito ao aspecto psicológico do paciente.

Para Moroni (1982), o paciente mutilado é aquele que sofreu uma deformidade ou deformação destruindo partes da face como olho, orelha, maxilar, nariz, sofrendo, muitas vezes, extensas mutilações faciais que comprometem o funcionamento fisiológico das partes afetadas. As malformaçóes congênitas ou 
traumatismos, sequelas pós-infecciosas, pós-cirúrgicas e pós-queimaduras, os tumores e suas consequências constituem os principais fatores etiológicos responsáveis pelas mutilaçóes faciais.

Nas crianças, a perda do globo ocular é causada em sua maioria por acidentes domésticos, segundo Centurion, oftalmologista e diretor do IMO (Instituto de Moléstias Oculares). Ele afirma que: "entre os agentes causadores, as estatísticas são variáveis, porém podemos inferir que os objetos pontiagudos, as contusóes e as substâncias cáusticas são as causas mais comuns [de perda ocular], em crianças” (Vinhal, 2007). Arcieri, Rocha, Resende e Machado (2004) demonstram haver um alto índice de traumas oculares em crianças que acontecem na própria casa da vítima. Diferentemente do adulto, a criança está em fase de desenvolvimento físico. A face, assim como os ossos, ainda se encontra em crescimento, sendo que qualquer agressão sofrida neste período pode, mais tarde, ocasionar deformidade de maior ou menor grau. Ao se tratar de perda ocular, ou anoftalmia congênita, o cérebro da criança recebe a mensagem da inexistência do órgão, tendendo a atrofiá-lo já que não possui função orgânica. E, caso não haja a reabilitação com a prótese ocular, o lado anoftálmico do rosto da criança interrompe seu crescimento, enquanto o lado são continua a desenvolver-se. Segundo Soares (1997):

a expansão orbitária, principalmente quando iniciada o mais precocemente possível, é o estímulo principal para o crescimento craniofacial, sendo por isso indispensável no tratamento da cavidade anoftálmica congênita [...]. Apesar do crescimento ósseo craniofacial estar concluído em torno dos 13 anos, estas cirurgias [enucleação e evisceração] não podem prescindir do implante quando feita após esta idade, pois a cavidade sem implante também sofre redução tardia da sua amplitude por expansão dos seios paraorbitários (Soares, 1997, p. 363-364).

Em adultos, os principais agentes causadores de perdas oculares são as infecções (Leão et al., 2010), o glaucoma (Goiato, Mancuso, Fernandes, \& Dekon, 2004) e os traumatismos (Goulart, Queiroz, Fernandes, \& Oliveira, 2011). Para esses pacientes, a prótese ocular apresenta função estética.

Pesquisas acerca dos pacientes em situação de prótese ocular sugerem necessidade de tratamento psicológico no decorrer do processo de adaptação da prótese. A perda do olho e, consequentemente, da visão pode implicar em sintomas psicológicos que, se não tratados, podem permanecer mesmo após a reabilitação facial. Botelho, Moura e Volpini (2003) investigaram 30 pacientes anoftálmicos com o intuito de avaliar os aspectos psicológicos decorrentes da perda do 
globo ocular, assim como os fatores interpessoais nela envolvidos, bem com a reintegração psicossocial desses pacientes. Eles verificaram baixa autoestima em $53 \%$ dos participantes e autoimagem distorcida em 37\% da amostra. Dentre os participantes, $37 \%$ apresentaram estado depressivo após a perda do globo ocular, sendo que $36 \%$ destes não superaram os sintomas mesmo após a colocação da prótese. Araújo, Cardoso e Morais (2007) verificaram, por sua vez, os principais sentimentos relatados por 41 pacientes anoftálmicos em relação a autopercepção, família, colegas de trabalho e pessoas estranhas. Dentre os sentimentos estão presentes a depressão $(17,8 \%$ da amostra), a tristeza $(16,4 \%)$, o conformismo $(13,7 \%)$ e o sentir-se diferente dos outros $(9,6 \%)$.

\section{Experienciaçáo e focalizaçáo: descriçáo e efetividade}

O conceito de experienciação surgiu a partir da parceria entre Rogers e Gendlin, com início na Universidade de Chicago em 1953. Os conceitos desenvolvidos por Rogers, como a tendência atualizante, as condiçóes necessárias para o psicoterapeuta, a conexão com a experiência interna em prol do crescimento pessoal, entre outros, além da fenomenologia e existencialismo, inspiraram Gendlin na construção de seu próprio pensamento, sistematizado na Teoria do Implícito (Gendlin, 1997).

O conceito de experienciação é definido a partir de uma concepção de corpo, que em Gendlin tem lugar de destaque. O corpo é mais do que o canal pelo qual as emoçóes, sentimentos e sensaçóes depositam os dados do mundo externo. Ele contém seu próprio conhecimento implícito e significado sentido, tornando-se um elemento ativo impulsionador da evolução da pessoa e seu crescimento (Barceló, 2008). A experienciação é definida por Gendlin como a interação dinâmica e indissociável entre manifestações fisiológicas, sentimentos, crenças e cogniçóes ocasionadas pelo corpo ativo (Gendlin, 1959). Nela, os processos internos do indivíduo ocorrem em uma única instância, sem primazia de conteúdo, pensamento ou emoção específica. Essa instância é uma sensação sentida (felt-sense) que carrega em si significados implícitos conscientemente sentidos, mas desconhecidos a priori (Barceló, 2008; Gendlin, 1984).

A focalização, por sua vez, está diretamente relacionada à experienciação. Ela é a ferramenta criada por Gendlin para facilitar o autoconhecimento a partir do contato com o felt-sense (Gendlin, 2006). O primeiro objetivo da focalização é acessar, explorar e explicitar o felt-sense de modo a gerar uma mudança de fluxo da qualidade de bloqueio para a qualidade de fluidez experiencial. Esse avanço ex- 
periencial (carrying forward) é acompanhado por uma mudança corporal de nível cognitivo/afetivo/comportamental (felt-shift) sentida em maior ou menor proporção, podendo representar um forte alívio tensional, lágrimas, risos, ou mesmo um pequeno conforto para seguir adiante (Gendlin, 1984). A focalização é, portanto, "a habilidade formalizada de um processo natural" (Barceló, 2010, p. 4) do qual Gendlin abstraiu o funcionamento e o transformou em passos possíveis de serem ensinados e praticados. $\mathrm{O}$ uso da ferramenta de focalização, em seu início, fazia-se restrito a sessóes terapêuticas, mas ao longo do tempo tornou-se independente, passando a estar presente em empresas, escolas, hospitais, etc (Gendlin, 2006).

$\mathrm{O}$ ato de focalizar é realizado em seis movimentos de um único processo. $\mathrm{O}$ primeiro deles denomina-se "clareando o espaço" e consiste em um relaxamento silencioso com o objetivo de voltar a atenção ao próprio corpo de modo a senti-lo e escutá-lo. Nessa etapa, o psicoterapeuta sugere ao cliente realizar perguntas sobre si mesmo e a situação específica a fim de detectar o problema ou situação que mais o incomoda ou o mobiliza naquele momento (Gendlin, 2006, p. 70). Detectado o ponto a ser focalizado, segue o segundo passo do processo: o felt-sense. Essa etapa tem como objetivo promover o contato do cliente com a sensação sentida daquela experienciação. Para isso, o psicoterapeuta sugere ao cliente distanciar-se da situaçáo focalizada e atentar apenas às sensaçóes corporais. O contato com o felt-sense é seguido pelo terceiro passo do processo, denominado "buscando um gancho", que objetiva encontrar a palavra, frase ou imagem que melhor se adéqua à sensação sentida. Em sequência, é necessário verificar se a característica encontrada para a sensaçáo sentida realmente engloba sua complexidade. Para o quarto passo, denominado "ressoando", é necessário que o cliente recapitule a sensaçáa sentida, que nem sempre permanece igual, e a compare novamente ao gancho encontrado. O felt-shift, ou mudança corporal de nível cognitivo/afetivo/ comportamental, é o indicativo dessa etapa, por determinar a exatidão entre o felt-sense e o gancho estabelecido (Hendricks, 2001, p.01). Caso não ocorra uma mudança corporal na quarta etapa, aplica-se o quinto passo: "perguntando". O objetivo dessa etapa consiste em uma nova investigação da sensação sentida e um posterior questionamento utilizando o gancho. Encontrado o gancho e obtido o felt-shift, o sexto e último passo é realizado: a "acolhida". Esta etapa consiste na aceitação pelo cliente dos processos vivenciados pelo seu corpo, seja ele um alívio, uma resposta a um problema prévio ou lágrimas. Acolher a mudança ocorrida mantém o corpo aberto às próximas experiências (Gendlin, 2006).

A respeito da efetividade da focalização e experienciação, a revisão de Hendricks (2001) e de Wagner (2006) mostra que a focalização é eficaz no desenvolvimento da experienciação, assim como se correlaciona positivamente com 
indicadores de sucesso terapêutico frente a problemas e distúrbios como psicose, depressão, vício em heroína e problemas de saúde.

Quanto à literatura nacional, uma busca nos bancos de dados Scielo, Periódicos Capes, Plataforma Lattes, Pepsic e Banco de Teses da Capes, com o emprego das palavras-chave focalização, experienciação, escala de experienciação e Gendlin, apontou a presença de dois artigos. O primeiro emprega a Escala de Experienciação na avaliaçáo de processos de psicoterapia de grupo (Gardini, 1996) e o segundo discute em nível teórico o impacto do conceito de experienciação na abordagem centrada na pessoa (Messias \& Cury, 2006).

\section{Objetivo do estudo}

A prótese ocular reconstitui a cavidade anoftálmica ausente de globo ocular proveniente de acidentes, alteraçóes patológicas ou congênitas. Seu objetivo principal é reconstruir esteticamente a face do paciente prejudicada pela malformação ou perda do globo ocular, além de proteger a cavidade anoftálmica contra infecções.

A anoftalmia ocular afeta o domínio psicológico dos pacientes. Além da perda do órgão e, consequentemente, da visão, os procedimentos médicos e cirúrgicos subsequentes e necessários para a possível reabilitação com a prótese ocular são bastante dolorosos (Soares, 1997). O nível em que o domínio psicológico pode ser afetado varia de acordo com o significado atribuído pela pessoa em funçấo de suas experiências anteriores e estilo de vida. Estudos acerca dos aspectos psicológicos de pacientes em processo de reabilitação facial indicam baixa autoestima, dificuldade de relacionamento interpessoal, imaturidade afetiva e dificuldade de relacionamento com o próprio corpo, além de maior passividade e dependência (Amaro, Belfort, \& Erwenne, 2000).

Esse contexto foi o gatilho para a motivação de intervir psicologicamente nesses pacientes de modo a atuar em seu desenvolvimento integral e não em seus sintomas imediatos. Para isso, foi selecionada uma teoria psicológica que norteasse esse objetivo: a Teoria do Implícito de Gendlin e seus conceitos de experienciação e focalização. $\mathrm{O}$ conceito de experienciação entrelaça os aspectos cognitivos, como percepção e pensamentos, aos aspectos afetivos ligados às emoçôes e sentimentos, assim como os interliga aos aspectos fisiológicos, como as sensações corporais. Há evidências de que a experienciação é um preditor de sucesso terapêutico (Hendricks, 2001). Já a focalização é uma ferramenta psicoterápica ou educativa (Gendlin, 2006) que possibilita ao cliente aproximar-se de 
sua própria experiência, direcionando-o aos processos internos de modo a que ele maneje melhor aquilo que o preocupa ou que interrompe seu fluxo processual (Barceló, 2008). A focalização atua diretamente na habilidade experiencial da pessoa, descrita em níveis pela Escala de Experienciação (Klein, Methieu, Gendlin, \& Kiesler, 1969).

Buscou-se aplicar a técnica psicoterápica de focalização em pacientes em situação de prótese ocular por meio de uma intervenção breve de quatro sessóes, de forma que o objetivo deste estudo consiste em avaliar a efetividade dessa ferramenta no aumento do nível de habilidade experiencial do cliente.

\section{Método}

Para avaliar os efeitos da psicoterapia breve, orientada pela focalização com pacientes em situação de prótese ocular, foi realizado um estudo com delineamento experimental de grupo caso-controle respeitando os critérios éticos de pesquisa e aprovação de comitê de ética da Universidade Federal de Minas Gerais, no 0487.0.203.000-10. Ambos os grupos, experimental e controle, responderam ao Teste de Habilidade Experiencial (THE), aplicado por estudantes de psicologia devidamente treinadas, antes e depois do processo de reabilitação facial com a prótese ocular. Após a testagem inicial, os participantes do grupo experimental vivenciaram encontros terapêuticos individuais guiados pela focalização, em sequência a cada etapa de confecção e adaptação da prótese ocular, totalizando quatro encontros terapêuticos. Já os participantes do grupo controle passaram pelo mesmo processo de reabilitação facial, sem as intervençóes psicológicas.

\section{Participantes}

Os participantes deste estudo compóem uma amostra de conveniência, seguindo critérios como faixa etária acima de seis anos, realização do processo padrão de reabilitação facial com a prótese ocular em quatro etapas, possibilidade de participar dos encontros terapêuticos após cada etapa do processo de reabilitação facial e aceitação em participar do estudo.

Para a obtenção da amostra do estudo, a proposta da pesquisa e o termo de consentimento livre e esclarecido eram apresentados por um funcionário treinado da Oculart a cada paciente em sua primeira consulta. Este, por sua vez, escolhia participar ou não do estudo. A alocação dos grupos experimental e controle buscou emparelhar, sempre que possível, os participantes a partir de 
suas características sociodemográficas como idade, sexo, escolaridade e residência (região metropolitana de Belo Horizonte e interior de Minas Gerais). Assim, os critérios de inclusão determinaram que os participantes tinham de possuir as seguintes características: (1) ser pacientes novos do laboratório Oculart, (2) ter mais de seis anos de idade, (3) realizar o processo padrão de reabilitação facial com a prótese ocular em quatro etapas, (4) ter a possibilidade de participar dos encontros terapêuticos após cada momento da reabilitaçáo facial. Na medida em que o laboratório Oculart recebe uma média de quatro novos pacientes por mês, provenientes de todo o estado de Minas Gerais, a amostra do estudo foi obtida por meio de seleção intermitente dos pacientes novos que ingressavam no tratamento de reabilitação facial.

Fizeram parte da amostra 18 pacientes do laboratório Oculart (Confecção e Adaptação de Prótese Ocular Ltda.), localizado no centro de Belo Horizonte. O grupo experimental foi composto por 10 participantes entre nove e 64 anos (m $=25,50 ; \mathrm{dp}=17,37)$, sendo $40,00 \%$ do sexo feminino. Do grupo experimental $70,00 \%$ residiam na região metropolitana de Belo Horizonte e 30,00\% no interior do Estado, 40,00\% relataram escolaridade em nível fundamental e 60,00\% em nível médio. Já o grupo controle foi formado por oito participantes entre $13 \mathrm{e}$ 69 anos ( $m=35,75 ; \mathrm{dp}=17,88$ ), sendo $37,50 \%$ do sexo feminino, $37,50 \% \mathrm{com}$ nível de escolaridade fundamental, 50,00\% nível médio e 12,50\% nível superior. Do grupo controle, 75,00\% eram do interior do Estado e 25,00\% residentes da região metropolitana de Belo Horizonte.

\section{Instrumentos}

Teste de Habilidade Experiencial (THE): visa mensurar o nível espontâneo de experienciação do respondente. Em cada um dos três itens do teste, o respondente deve imaginar e vivenciar cenas específicas, inicialmente descritas no enunciado de cada item. Quando o respondente começa a vivenciar a cena descrita, perguntas referentes às dimensôes fundamentais da experienciação são feitas pelo examinador, envolvendo sensaçôes corporais, sentimentos, reaçôes e pensamentos. A aplicação do teste é feita de modo individual, sem tempo limite e em pessoas com idade acima de seis anos. Não há pré-requisito de escolaridade para a aplicação do THE. A fala do respondente no decorrer da aplicação do teste é gravada e, posteriormente, transcrita. O conjunto de respostas fornecido pelo indivíduo frente a cada item é classificado em níveis experienciais, seguindo o Método de Análise e Classificação de Níveis Experienciais (MACNE), utilizado para a formação dos escores. Náo há estudos prévios publicados sobre 
a validade e confiabilidade do THE. No entanto, em relação aos dados deste estudo, o teste mostrou boa consistência interna, com alfa de Cronbach de 0,90 e carga fatorial igual ou superior a 0,90 dos três itens do teste junto a um fator identificado através do procedimento de análise fatorial exploratória da matriz de correlação policórica. A análise fatorial exploratória foi realizada no software Mplus 5.2.

\section{Coleta e análise de dados}

A coleta de dados deste estudo realizou-se na Oculart - Confecção e Adaptação de Prótese Ocular Ltda, nos anos de 2010 a 2012, por uma psicóloga com formação em psicoterapia humanista e duas alunas do grupo de pesquisa do Laboratório de Investigação Cognitiva da Universidade Federal de Minas Gerais (LAICO). As voluntárias foram devidamente treinadas na utilização do Teste de Habilidade Experiencial (THE).

O THE foi aplicado de modo individual, com gravação e transcrição das falas dos respondentes em duas fases distintas, sendo a primeira no início do processo de reabilitação facial e a segunda ao final do mesmo processo. O grupo experimental passou por quatro encontros terapêuticos guiados pela focalização e realizados pela pesquisadora, diferentemente do grupo controle, submetido apenas ao teste/re-teste. Visando investigar a efetividade da focalização, avaliou-se o tamanho do efeito da diferença de crescimento da média do grupo experimental e do grupo controle. O cálculo do tamanho do efeito utilizado foi o de Cohen (1988), corrigido o viés de tamanho de amostra por Hedges e Olkin (1985). A fórmula descrita a seguir:

$$
d=m g e-m g c / \sqrt{\frac{d p g e^{2} *(N g e-1)+d p g c^{2} *(N g c-1)}{N g e+N g c-2}}
$$

refere-se à diferença entre a média do grupo experimental (mge) e a média do grupo controle $(\mathrm{mgc})$, dividida pela raiz quadrada do desvio amostral reunido:

$$
\sqrt{\frac{d p g e^{2} *(N g e-1)+d p g c^{2} *(N g c-1)}{N g e+N g c-2}}
$$


sendo dpge o desvio padrão do grupo experimental, Nge o tamanho amostral do grupo experimental, dpgc o desvio padrão do grupo controle e $\mathrm{Ngc}$ o tamanho amostral do grupo controle.

Cohen, citado por Thompson (2008), considera o valor de 0,2 um indicador de tamanho de efeito pequeno, o valor de 0,5 um indicador de tamanho médio de efeito e o valor de 0,8 e acima, um indicador de tamanho grande de efeito. Este mesmo critério é utilizado neste artigo.

\section{Resultados}

A variável idade apresentou distribuição normal $(\mathrm{m}=30,06 ; \mathrm{dp}=17,85$; Kolmogorov-Smirnov $(\mathrm{KS}-\mathrm{Z})=0,75 ; \mathrm{p}=0,63)$. As demais variáveis não apresentaram distribuição normal: escolaridade $(\mathrm{m}=1,67 ; \mathrm{dp}=0,59 ; \mathrm{KS}-\mathrm{Z}=1,37 ; \mathrm{p}=$ $0,05)$, sexo $(m=0,39 ; d p=0,50 ; K S-Z=1,66 ; p=0,01)$ e residência $(m=1,50$; $\mathrm{dp}=0,51 ; \mathrm{KS}-\mathrm{Z}=1,42 ; \mathrm{p}=0,04)$.

Os grupos experimental e controle não apresentaram diferenças estatisticamente significativas no que tange a faixa etária $(t=1,23 ; \mathrm{gl}=16 ; \mathrm{p}=0,24)$, sexo $(Z=-0,11 ; p=0,92)$, escolaridade $(Z=-0,41 ; p=0,69)$ e residência $(Z=-1,84$; $\mathrm{p}=0,06)$. No pré-teste, o escore médio do grupo experimental foi de 3,03 ( $\mathrm{dp}=$ $0,10)$, e o escore médio do grupo controle foi de 3,00 $(\mathrm{dp}=0,00)$. No pós-teste, o escore médio do grupo experimental foi de $3,13(\mathrm{dp}=0,32)$ e o grupo controle permaneceu com escore médio de 3,00 $(\mathrm{dp}=0,00)$. A diferença entre os escores médios no pré-teste/pós-teste para o grupo experimental foi de $0,10(\mathrm{dp}=0,35)$ e para o grupo controle, de $0,00(\mathrm{dp}=0,00)$. O resultado do tamanho do efeito foi de 0,38 , com intervalo de $-0,58$ a $1,30(\mathrm{p}=0,44)$.

\section{Discussão}

Os resultados do estudo indicaram um efeito pequeno-moderado da intervenção junto ao desenvolvimento da habilidade experiencial dos pacientes. O efeito encontrado não apresentou significância estatística. Isso indica que o efeito é passível de ser considerado, mas é uma evidência fraca, pois a probabilidade de que seja devido ao acaso é relativamente grande $(\mathrm{p}=0.44)$. Mantendo as mesmas características de média e desvio padrão, o $\mathrm{N}$ necessário seria de 50 , tanto no grupo controle quanto no grupo experimental, para que a diferença fosse estatisticamente significante. Com a amostra atual, seria necessário um tamanho de efeito de 1,02 para que a diferença entre os grupos fosse estatisticamente significativa. 
Apesar de o efeito ter sido pequeno-moderado, dois resultados mostraram indícios favoráveis à mudança. $\mathrm{O}$ primeiro deles foi o aumento do desvio-padrão do grupo experimental do pré $(\mathrm{dp}=0,10)$ para o pós-teste $(\mathrm{dp}=0,32)$. Mesmo não tendo gerado um efeito forte, a intervenção provocou um estado inicial de mudança no que tange a esse aumento da variância intragrupo. O segundo resultado, por sua vez, diz respeito à mudança experiencial de nível três para nível quatro em um dos participantes do grupo experimental. Para esse paciente, a intervenção foi efetiva na mudança de um padrão externalista para internalista. Considerando que o perfil experiencial de cada participante do grupo controle foi de nível três, sem nenhuma variação nas respostas apresentadas, o caso de mudança encontrada no grupo experimental passa a ser qualitativamente relevante.

O resultado do pré-teste para ambos os grupos mostrou a predominância quase absoluta de foco de referência externalista. Isso indica que os pacientes atribuíam ao ambiente a causa de seus sentimentos, emoções e cogniçóes. Essa característica se manteve nos participantes do grupo controle no pós-teste, de forma muito homogênea, a ponto de não apresentar nenhum desvio-padrão no perfil de respostas. No grupo experimental, apesar de o padrão espontâneo de nível três ter se mantido fortemente, a psicóloga que realizou as intervençóes relatou que todos os participantes deste grupo atingiram o felt-sense ou nível experiencial cinco em pelo menos uma das quatro sessóes. Essa ocorrência mostra que o padrão espontâneo é alterado no contexto de suporte da focalização, que mobiliza níveis experienciais não necessariamente usuais e recorrentes da pessoa. Em uma mesma sessão psicoterápica, a focalização provoca a ocorrência de vários níveis experienciais, demarcando a geração de ciclos de variações. Um futuro estudo envolve a análise do ciclo dos níveis experienciais vivenciados pela pessoa ao longo das sessóes, ou seja, em contexto de suporte, e sua relação com a geração de mudanças no nível experiencial espontâneo.

Além das mudanças momentâneas de nível experiencial, identificadas em contexto de suporte terapêutico, a psicóloga que realizou as intervençóes também relatou que todos os participantes do grupo experimental demonstraram sentimentos como alívio, bem-estar, leveza e tranquilidade, indicando que as sessôes possivelmente geraram também mudanças momentâneas no bem-estar dos pacientes. No entanto, esse estudo centrou seu foco na produção de mudanças estruturais capazes de gerar alteraçóes de nível espontâneo de experienciação. Para que uma terapia seja efetiva, é importante que as mudanças não ocorram apenas em níveis de suporte, mas que sejam geradas espontaneamente pela pessoa, propiciando autonomia. 
Os resultados discutidos englobam algumas limitaçóes. A primeira envolve o pequeno tamanho da amostra, aumentando muito o intervalo de confiança do efeito da intervenção. Apesar desse limitador, a amostra utilizada é relativamente representativa para a população em situação de prótese ocular por ser proveniente de uma das duas clínicas que realizam a confecção e adaptação da prótese ocular no estado de Minas Gerais. Uma segunda limitaçáo envolve a falta de outros critérios de sucesso psicoterápico, tais como autoestima e qualidade de vida. Um estudo futuro pode incorporar essas variáveis, além do nível de experienciaçáo.

Este estudo pretendeu contribuir com implicaçôes para a prática clínica e a literatura no campo da experienciação. Foi aplicado um desenho de caso-controle e a análise do tamanho do efeito para a técnica de focalização. A focalização foi articulada em um contexto de psicoterapia breve. $\mathrm{O}$ estudo abre perspectivas de intervenção com a focalização em contextos variados que requerem psicoterapia breve como é o caso de pacientes oncológicos, diabéticos, em situação de hemodiálise, além de outros contextos, como, por exemplo, estudantes em preparação para o ingresso na universidade, etc. Em relaçáo à literatura nacional, este estudo abrangeu um campo ainda pouco explorado no Brasil, o da focalizaçáo, abrindo possibilidades de pesquisa desta técnica para a psicoterapia em contextos brasileiros.

\section{Referências}

Amaro, T. A. C., Belfort, R., \& Erwenne, C. M. (2000). Estudo psicológico de pacientes enucleados por trauma ou tumor ocular em uso de prótese. Acta Oncológica Brasileira, 20(4), 138-142.

Araújo, P., Cardoso M., \& Morais, S. L. (2007). Implicaçóes psicossociais em pacientes com perda do globo ocular. Revista Cirurgia Traumatologia Bucomaxilo-facial, 7(1), 79-84.

Arcieri, E. S., Rocha, S. J., Resende, A. L., \& Machado, R. P. (2004). Trauma ocular em crianças: um estudo epidemiológico na Universidade Federal de Uberlândia nos últimos cinco anos. Revista Médica de Minas Gerais, 14(1), 13-17.

Barceló, T. (2008). Focusing I y II: Escuchar el propio cuerpo. Proceso y técnica del enfoque corporal. Espanha: Instituto de Formación Ramon Serra.

Barceló, T. (2010). Filosofia experiencial y focusing. Espanha: Instituto de Formación Ramon Serra.

Botelho, N., Moura, A. E., \& Volpini, M. (2003). Aspectos psicológicos em usuário de prótese ocular. Arquivo Brasileiro de Oftalmologia, 66, 637-643.

Cohen, J. (1988). Statistical power analysis for the behavioral sciences. Hillsdale, NJ: Lawrence Erlbaum Associates. 
Fonseca, E. (1987). Prótese ocular. São Paulo: Panamed.

Gardini, R. C. (1996). Avaliação do processo de psicoterapia de grupo na escala Gendlin e Tomlinson. Mudanças, 2(2), 113-128.

Gendlin, E. T. (1959). The concept of congruence reformulated in terms of experiencing. Counseling Center Discussion Papers, 5(12). Recuperado em 23 de outubro, 2011, de <http://www.focusing.org/gendlin/docs/gol_2077.html>.

Gendlin, E. T. (1984). The politics of giving therapy away: Listening and focusing. In D. Larson (Ed.), Teaching psychological skills: models for giving psychology away (pp. 287-305). Monterey: Brooks/Cole. Recuperado em 18 de julho, 2011, de <http://www.focusing.org/ gendlin/docs/gol_2056.html>.

Gendlin, E. T. (1997). A process model. New York: The Focusing Institute. Recuperado em 05 de julho, 2011, de <http://www.focusing.org/gendlin/docs/gol_2161.html>.

Gendlin, E. T. (2006). Focalizaçấo: via de acesso a sabedoria corporal. São Paulo: Gaia.

Goiato, M. G., Mancuso, D. N., Fernandes, A. R., \& Dekon, F. C. (2004). Estudo sobre as causas mais freqüentes de perdas oculares. Arquivos Odontológicos, 40(3), 271-276.

Goulart, D. R, Queiroz, E., Fernandes A. R., \& Oliveira, L. M. (2011). Aspectos psicossociais envolvidos na reabilitação de pacientes com cavidade anoftálmica: implicaçóes do uso de prótese ocular. Arquivo Brasileiro de Oftalmologia, 75(5), 330-334.

Hedges, L. V., \& Olkin, I. (1985). Statistical methods for meta-analysis. Orlando, FL: Academic Press.

Hendricks, M. N. (2001). Focusing-Oriented/ Experiential Psychotherapy: Research and practice. In D. Cain \& J. Seeman (Eds.). Humanistic psychotherapy handbook of research and practice (pp. 221-252). Washington, DC: American Psychological Association.

Klein, M. H., Mathieu, P. L., Gendlin, E. T., \& Kiesler, D. J. (1969). The experiencing scale: A research and training manual. Wisconsin: Wisconsin Psychiatric Institute.

Leão, M. R. R., Junqueira, D. L. M., Junior, A. F., Navarro, R. M., Contrera, J. C., \& Miyashita, D. (2010). Análise dos casos de perda do globo ocular no Hospital Regional de Presidente Prudente no periodo de 2008 a 2010. Recuperado em 12 de dezembro, 2011, de <http:// oftalmologiausp.com.br/ePoster/PDF/i24.pdf>.

Messias, J. C., \& Cury, V. (2006). Psicoterapia Centrada na Pessoa e o impacto do conceito de experienciação. Psicologia: Reflexão e Crítica, 19(3), 355- 361.

Moroni, P. (1982). Reabilitação buco-facial: cirurgia e prótese. São Paulo: Panamed.

Resende, J. (1997). Fundamentos da prótese buco-maxilo-facial. São Paulo: Sarvier.

Soares, E. (1997). Cirurgia plástica ocular. São Paulo: Roca.

Thompson, B. (2008). Foundations of behavioral statistics: an insight-based approach. New York: The Guilford Press.

Vinhal, M. (2007). Trauma ocular infantil: é possível prevenir os acidentes que envolvem os olhos das crianças? (Entrevista). Recuperado em 08 setembro, 2008, de <http://www. 
revistavigor.com.br/2007/04/25/trauma-ocular-infantil-e-possivel-prevenir-os-acidentes-que-envolvem-os-olhos-das-criancas $>$.

Wagner, K. (2006). Inside out: focusing as therapeutic modality. Journal of Humanistic Counseling, Education and Development, 45, 45-59.

Recebido em 16 de abril de 2012

Aceito para publicação em 24 de outubro de 2012 